\title{
REVIEW
}

\section{Viral Respiratory Infections in Hematological Patients}

\author{
Giovanni Gabutti (D) - Francesco De Motoli - Federica Sandri •
}

Maria Vittoria Toffoletto · Armando Stefanati

Received: March 31, 2020 / Published online: July 7, 2020

(c) The Author(s) 2020

\section{ABSTRACT}

Viral infections of the respiratory system represent one of the most important complications in hematological patients in terms of both the severity of the clinical picture and its related impact on the duration of hospitalization, and of mortality. The most implicated viruses are those that commonly cause community-based respiratory diseases: respiratory syncytial virus, Influenza virus and rhinovirus. However, in some cases the clinical picture may be triggered by first infection with or reactivation of pathogens normally not responsible for clinically relevant diseases in immunocompetent subjects. This issue is currently being taken into

Enhanced Digital Features To view digital features for this article go to https://doi.org/10.6084/m9.figshare. 12497915.

G. Gabutti $(\bowtie) \cdot$ A. Stefanati

Department of Medical Sciences, University of Ferrara, Ferrara, Italy

e-mail: giovanni.gabutti@unife.it

F. De Motoli · F. Sandri - M. V. Toffoletto Post-Graduate School of Hygiene and Preventive

Medicine, University of Ferrara, Ferrara, Italy greater consideration within the scientific community. However, the strong heterogeneity in the epidemiology and clinical expression of these infections and the lack of adequate therapeutic options imply that there is currently no uniform consensus on the best management of these patients. The main purpose of this review is to highlight which viruses are currently most implicated in the onset of these infections, what is their incidence in so heterogeneous and fragile patients and the factors that lead to disease's onset and evolution. Possible or available clinical management options, diagnostic and therapeutic tools, and preventive and prophylaxis measures are also discussed.

Keywords: Cancer; Hematological malignancy; Immunocompromised; Respiratory viral infections; Stem cell transplantation 


\section{Key Summary Points}

Respiratory viral infections represent one of the most feared complications in the hematological patient in terms of both clinical severity and mortality.

Influenza (IFV) and parainfluenza viruses (PIV), respiratory syncytial virus (RSV), adenovirus (AdV), rhinovirus (RhV) and coronavirus $(\mathrm{CoV})$ are the most relevant agents involved in respiratory infections.

Respiratory infections in immunocompromised patients have an initial involvement of the upper respiratory tract; the feared progression to the lower respiratory tract is strictly related to clinical conditions of patients as well as several risk factors.

Defining effective therapeutic and prophylactic strategies is challenging, especially considering the variability spectrum of onco-hematological patients and the variety of respiratory viruses involved.

Although the scientific community recognizes the relevance of these infections, there is no uniform consensus on available clinical management options, diagnostic and therapeutic tools, and preventive and prophylaxis measures.

\section{BACKGROUND}

Infections are among the most important complications in subjects affected by cancer and/or in recipients of hematopoietic stem cell transplantation (HSCT) and often endanger the life of the immunosuppressed patient [1]. Infections affecting the respiratory system are particularly frequent in this population, and those involving the lower respiratory tract (LRTI) are often related to the highest severity and mortality [1]. Among the various etiological agents, community-acquired respiratory viruses (CRVs) represent a relevant cause in terms of morbidity and mortality among patients with hematological malignancies [2].

On one hand, the progress of therapeutic interventions has led to an increase in the survival and recovery of hematological patients; on the other, the wide use of chemotherapy and immunosuppressive treatments has further increased the infective risk for these patients [3].

Although attempts have been made to standardize the clinical and therapeutic approach to infective complications in immunocompromised hematological patients, the clinical expression of infections is extremely variable. The continuous changes in the epidemiology of infections, clinical manifestations of hematological patients and available therapies imply a lack of an uniform consensus on the best management of infective complications and the need for continuous updates [3]. Besides, the optimal approach to infective complications often needs to be personalized.

The reduced availability of easy and specific diagnostic methods has long hindered the feasibility of epidemiological studies on this issue; the advent of biomolecular tests has made it possible to obtain timely and accurate diagnosis of viral infections [2].

The exponential increase in interest in community-acquired viruses has been related to a growing number of scientific papers evaluating their role in the field of respiratory diseases in immunosuppressed patients [2].

From these premises, it is clear that infections in general still represent a frequent complication, and in some cases the main cause of death in these patients; it is therefore important to establish methods to prevent these problems [4]. In this review we will deal with respiratory viral infections in pediatric and adult hematological patients, describing epidemiological aspects, available diagnostic tools, treatment options and preventive measures. This article is based on previously conducted studies and does not contain any studies with human participants or animals performed by any of the authors. 


\section{RESPIRATORY VIRAL INFECTIONS IN HEMATOLOGICAL PATIENTS}

\section{Main Viruses and Pathogenesis}

Many relevant and well-known viral agents are involved in the onset of respiratory diseases in hematological patients such as influenza (Flu) and parainfluenza virus (PiV), respiratory syncytial virus (RSV), adenovirus (hAdV), rhinovirus (hRV) and coronavirus (CoV) as well as lesser known ones such as human metapneumovirus (hMPV) and bocavirus (hBoV) [2].

Other very well known pathogens such as herpes viruses [cytomegalovirus (CMV), Epstein-Barr virus (EBV), varicella-zoster virus (VZV), herpes simplex 1 and 2 (HSV1-2) and herpesvirus-6] frequently cause serious diseases in these patients but rarely affect the respiratory system. These diseases can follow a first contact with the virus or a viral reactivation related to immunosuppressive conditions [3, 4].

RSV is a single-stranded RNA virus belonging to the Paramyxoviridae family. Its genome can code for 11 proteins, including $G$ and $F$ proteins, important in the initial stages of infection; these proteins allow the virus to bind and penetrate within the epithelial cells of the upper airways [5].

The human influenza virus belongs to the Orthomyxoviridae family, and its genome is characterized by a single-stranded RNA segment. Its surface proteins, hemagglutinin (HA) and neuroaminidase (NA), play a fundamental role in the pathogenesis of infection and allow for the identification of different subtypes $[2,5]$. Three influenza virus genera are identified (A, B, C); influenza A virus is divided into different subtypes on the basis of the antigenic drift and shift mechanisms, which lead to minor or major changes in the composition of HA and NA, respectively [6].

Rhinoviruses (hRV) belong to the Picornaviridae family. They have a single-stranded RNA segment and are divided in three species (A, B and C) according to genomic and capsid characteristics.[5, 6]. To date, $>100$ serotypes are known [5]; $90 \%$ of these use the same receptor protein to penetrate the epithelial cells (I-CAM 1) [7].

Parainfluenza viruses (PiV) and metapneumovirus (hMPV) belong to the Paramyxoviridae family and have a single-stranded RNA segment. The PiV genome encodes six proteins: among these, hemagglutinin-neuraminidase (HN) and fusion protein $\mathrm{F}$ allow the virus to bind to the host cell and to merge with it. To date, five serotypes are known, which differ from each other from an epidemiological and clinical point of view.

hMPV, like RSV, binds to the host cell via a G protein and merges with it via an F protein; two hMPV subtypes are known (A and B, further divided into two subgroups: A1/A2, B1/B2) [5].

Coronaviruses $(\mathrm{CoV})$ are capsulated, singlestranded RNA viruses belonging to the Coronaviridae family. Although they are a common cause of colds in the general population, limited information is available in immunocompromised patients [5].

Adenoviruses (hAdV) are double-stranded, non-capsulated DNA viruses consisting of $>60$ serotypes divided into 7 species or subgroups $(A-G)$. The various serotypes can be associated with different clinical manifestations according to different receptor tropisms in different tissues [6].

Bocavirus (hBoV) is a small DNA virus belonging to the Parvoviridae family [6]. Currently, four hBoV variants are known: HBoV1, HBoV2, HBoV3 and HBoV4. HBoV1 was mainly isolated in LRTI, often associated with other pathogens, unlike the other three that generally cause gastroenteritis.

Respiratory viruses can be transmitted by direct contact and by inhalation of Flugge droplets or fomites. Capsulated viruses can resist in fomites for 2-72 h [6].

The $\mathrm{T}$ cell-mediated immune response is fundamental to protecting humans from viral infections. As this mechanism may be lacking in patients who undergo stem cell transplantation or who undergo immunosuppressive therapies, hematological patients are particularly susceptible to viral infections and to related complications [8]. 


\section{Risk Factors}

The clinical conditions of hematological patients, together with several risk factors, predispose patients to the onset of respiratory infections involving both the upper (URTI) and lower respiratory tract (LRTI).

In pediatric patients, a systematic review conducted in 2015 identified eight risk factors significantly associated with the development of LRTI infection in children: prematurity, low birth weight, male gender, having siblings, maternal smoking, history of atopy, lack of breastfeeding and crowding [9].

Advanced age, lymphopenia and recent allogenic stem cell transplantation have been recognized as general risk factors for the development of RSV lower respiratory tract infection [10]. In particular, HSCT patients may be at risk of CMV reactivation, an infection that can result in multi-organ involvement and in an increased risk of graft-versus-host disease (GVHD) [9].

In candidates for hematopoietic stem cell transplantation, a positive anamnesis for smoking, conditioning regimen with total highdose body irradiation and absolute lymphocyte count $<100 \mathrm{~mm}^{3}$ are significantly related to a risk of progression [11]. In addition, corticosteroid therapy administered at the onset of URTI is associated with a greater progression to LRTI [10].

The following risk factors for symptomatic progression to LRTI are known for RSV and PiV infections: advanced age, history of smoking, allogeneic HSCT, myeloablative regimen, neutropenia, lymphocytopenia, non-correspondent or unrelated donor transplant, graft-versus-host disease (GVHD), pre-engraftment status or early post-transplant period, and systemic use of corticosteroids [8].

The progression of influenza virus to LRTI implies lymphopenia, the use of steroids and the delayed start of antiviral therapy [11].

In patients with coronavirus infection, the presence of co-pathogens such as RSV and hostrelated factors such as age $<5$ years and being immunocompromised are factors that can contribute to LRTI progression [11].

\section{Outline of Clinical Aspects}

The severity of respiratory infections in immunocompromised patients is greater than that observed in immunocompetent subjects, although the clinical picture is substantially similar.

Diseases caused by these viruses vary from URTIs, usually self-limiting, to LRTIs, which are potentially lethal. The clinical evolution depends, as already stated, on the condition of the patient as well as the intrinsic virulence of the virus.

At onset, reported symptoms are therefore unspecific and related to an initial involvement of the upper respiratory tract: cough, rhinorrhea, otitis media, wheezing, and sore throat with or without fever $[4,12]$.

Other less frequently observed symptoms are diarrhea or gastrointestinal symptoms and conjunctivitis [9]. In other cases, as in influenza virus infection, the typical manifestations may be absent [2]. Dyspnea, hypoxemia and possible lung infiltrates may appear on chest x-ray with progression to LRTI [8]. Neutropenia is not a pivotal clinical fact but is rather a condition that predisposes patients to bacterial infections [4].

\section{Epidemiology}

The estimated incidence of the above-mentioned respiratory viruses has a wide range due to the variability in screening and diagnostic methods as well as study design. In hematological and HSCT patients, the trend and seasonal peak roughly reflect the classic behavior of these viruses in the population, although there are possibilities for significant variations in terms of incidence and mortality year by year [6].

Epidemic RSV outbreaks typically occur in winter like for influenza, except in tropical areas where the latter can occur throughout the year. Parainfluenza viruses generally spread all year round, with peaks in spring-summer, a period in which a greater number of hMPV cases are also observed [2]. 
hRV, RSV, PiV, hAdV and hMPV are the most frequently isolated viruses in myeloma patients, who generally have the highest median age. Seasonal peaks have been observed in winter and spring, except for $\mathrm{PiV}$, which peaks in summer [13].

In pediatric subjects, $\mathrm{hRV}$ is the main cause of respiratory infection compared to RSV and $\mathrm{PiV}[4,9,14]$. Also in this category of patients, a prevalent seasonality in winter $(31.5 \%)$ and spring (26.5\%) has been observed [9].

Marinelli et al. estimate that, in patients treated with autologous stem cell transplantation, hRV is the most common community-acquired virus (47\%) followed by $\mathrm{PiV}$ (14.1\%), influenza (11.2\%), RSV (11.8\%) and hMPV $(8.8 \%)$ [8]. Viruses that most frequently cause respiratory infections in the HSCT candidate patient include: RSV, AdV, influenza, PIV, hMPV, RhV, CoV and hBoV [15].

\section{Respiratory Syncytial Virus (RSV)}

Respiratory syncytial virus is the most important causative agent of LRTI in infants and children worldwide [16, 17]. Many children develop a primary infection within the first 2 years of life, but despite this permanent immunity is not acquired [18]. A Finnish study, evaluating serological IgG levels, showed that $37 \%$ of children had contracted an RSV infection in the first year of life, $68 \%$ within 2 years and $86 \%$ within 3 years of age. At least a third of these children then develop reinfection in subsequent years, confirming the fact that immunity in the first years of life is only partially protective [19]. The incidence rate of RSV infection in adult and pediatric hematologic subjects ranges between 1 and 50\% (Table 1) [6]. This value ranges between 1 and 12\% taking into account only adults [20] (Table 2). Although the mortality rate for pneumonia in healthy children and adults is low (0.5\%), this

Table 1 Incidence, mortality, preventive and therapeutic options against main respiratory infections in hematological patients. Modified from $[6,32]$

\begin{tabular}{lllll}
\hline Virus & $\begin{array}{l}\text { Incidence } \\
(\%)\end{array}$ & $\begin{array}{l}\text { Mortality } \\
(\%)\end{array}$ & Preventive options & $\begin{array}{l}\text { Therapeutic } \\
\text { options }\end{array}$ \\
\hline $\begin{array}{l}\text { Respiratory syncitial } \\
\text { virus }\end{array}$ & $1-50$ & $11-47$ & S, C, D & Ribavirin aerosol \\
Influenza & $1.3-40$ & $8-28$ & S, D & Oseltamivir \\
& & & Seasonal influenza immunization & ND \\
Rhinovirus & $2-34$ & $<5-41$ & S, D & ND \\
Metapneumovirus & $2-11$ & $6-40$ & S, C & Ribavirin \\
Parainfluenza virus & $3-27$ & $10-50$ & S, A, D & Cidofovir \\
& & & oseltamivir & ND
\end{tabular}

$A$ airborne precautions, $C$ contact precautions, $D$ droplet precautions, $S$ standard precautions 
Table 2 Incidence of the most common respiratory virus infections in children and adults with hematological malignancy

\begin{tabular}{llll}
\hline & & Hematological patients & \\
\cline { 3 - 4 } & & Children & Adults \\
\hline Respiratory syncytial virus & HM & - & $1-12 \%[20]$ \\
& ALL & $14-31 \%[22]$ & - \\
AML & $0.3-2.2 \%[22]$ & - \\
Lymphoma & - & - \\
Rhinovirus & Myeloma & - & $40-60 \%[13]$ \\
& Other tumors & $8.7 \%[23]$ & - \\
Influenza & HM & $33.0 \%[12]$ & $40 \%[33]$ \\
Parainfluenza virus & Leukemia & $33.9 \%[12]$ & - \\
Other tumors & $31.8 \%[12]$ & - \\
Human metapneumovirus & HM & - & - \\
& ALL & $36-38 \%[5,22]$ & - \\
& HM & $10 \%[39]$ & $2-7 \%[20]$ \\
& ALL & $18 \%[22]$ & -
\end{tabular}

$H M$ hematological malignancy not otherwise specified, $A L L$ acute lymphoid leukemia, $A M L$ acute myeloid leukemia

can reach $60 \%$ in immunocompromised untreated children and adults [17].

The impact of RSV on immunocompromised patients with myeloma following stem cell or solid organ transplantation is well known, being associated in $40-60 \%$ of cases with a progression from the upper to the lower respiratory tract $[13,21]$. Mortality can reach $30 \%$ after developing pneumonia [13].

Morbidity and mortality from this infection increase in premature babies, and in children with underlying heart disease or severe immunosuppression [16]. A single-center study [22] found that RSV was the second most common cause of respiratory viral infection accounting for $31 \%$ of all episodes of respiratory viral infections in children with acute lymphoblastic leukemia (ALL).

RSV infections occur in $0.3-2.2 \%$ of pediatric patients with acute myeloid leukemia, $14 \%$ of children with ALL [22] and $8.7 \%$ of children with various malignant diseases [23].
In a prospective study conducted in Athens on children with hematological malignancies (65\% of cases) or solid tumors who had received chemotherapy or HSCT treatment, the viral genome was isolated in $38.4 \%$ of subjects with URTI or LRTI infections, both febrile and nonfebrile. RSV was the most frequently isolated virus ( $46 \%$ of cases) followed by parainfluenza virus and bocavirus, identified in approximately $13 \%$ of children. Approximately $40 \%$ of children tested positive for a viral agent had a diagnosis of ALL, $11 \%$ of AML and 1\% of lymphoma, respectively [1].

\section{Influenza Virus (FLU)}

The influenza virus is probably the most studied and known of the community-acquired viruses.

Several countries, including Germany, have carried out seroprevalence studies on their population with regard to influenza $\mathrm{A}$ and $\mathrm{B}$ 
viruses: a prevalence of antibody levels (IgG) against viruses $\mathrm{A}$ and $\mathrm{B}$ has been observed in $87 \%$ and $47 \%$ of $0-17$-year-old subjects, respectively. In adulthood, the antibody prevalence is around $96 \%$ for virus A and $98 \%$ for B, with a decreasing trend for antibodies against virus A with aging. Vaccinated children have a significantly higher antibody level than unvaccinated ones [24]. Differently, a cross-sectional study performed in the United Arab Emirates showed a seroprevalence in the pediatric population quite similar to the one observed in the German population for B virus, but significantly lower for A virus [25].

Several monocentric studies have evaluated the global impact of epidemics on hematological patients: mortality appears to be similar to that of the general population although the durations of hospitalization and antiviral treatment appear be longer for hematological patients [2].

A retrospective study, performed in two cancer centers in the USA and Mexico on hematological patients hospitalized for influenza symptoms between 2009 and 2014 (during the AH1N1 pandemic and post-pandemic period), highlighted that the mean age of the patients was 49 (range 1-88) years and that the majority of patients had leukemia (54\%). Besides, $42 \%$ of hospitalized patients developed LRTI and 16\% died within 2 months of diagnosis [26].

Two prospective European surveys, probably considering only the most severe cases, estimated the mortality linked to the 2009 AH1N1 pandemic in HSCT patients to be $6.3 \%$ [2]. The incidence of influenza in hematological and/or transplant patients varies, according to the study, from very low to medium; nonetheless, the aforementioned virus remains a significant cause of morbidity and mortality [6] (Table 1). Nichols et al. report that the incidence in HSCT patients ranges between 1 and 3\% [27].

Influenza is the most common virus in ALL pediatric patients, making up about 36\% [22] and $38 \%$ [5] of respiratory infections.

Notaby, influenza is highly variable in terms of both genome (antigenic drift, shift and recombination) and virulence; this variability can have a significant impact in immunosuppressed individuals (due to treatment or transplant regimens), with an increased risk of complications and death [28].

In patients with myeloma, influenza is the respiratory virus associated with the greatest morbidity and mortality, and their risk of developing influenza is approximately six times higher than in controls [10].

The increase in mortality due to this infection is related to the onset of respiratory complications, bacterial super-infections or the need for mechanical ventilation [5].

Disease progression to LRTI occurs in up to $35 \%$, and mortality ranges between 15 and $28 \%$ in patients with pneumonia. Therefore, early treatment is recommended within 2 days after the onset of symptoms [11].

\section{Human Rhinovirus (hRV)}

hRV circulates throughout the year and is the most common cause of URTI, being responsible for around $52-79 \%$ of cases of colds. The incidence of infection in hematological patients is estimated between 2 and 34\% [6]. Although it causes a benign disease in immunocompetent patients, its role in terms of morbidity and mortality in at-risk subjects has only recently been highlighted [2].

In children with hematological malignancies and/or HSCT, hRV was found in $23-62 \%$ of URTI [29-32] and $65 \%$ of LRTI [14]. In this last work, the mean age of children was 6.9 years, and half of the patients affected by a respiratory virus had a diagnosis of leukemia or lymphoma (LL). hRV was the most detected virus in all the patients recruited in the study (hematological, with solid tumor or post-HSCT). In patients affected by leukemia or lymphoma and by a viral infection, hRV was detected in $65 \%$ of URTI and 59\% of LRTI, while in children the virus represented $77 \%$ of URTI and $50 \%$ of LRTI in the post-HSCT period [14].

The estimated mortality rate ranges between 5 and $41 \%$, also according to host factors [6].

A retrospective study showed that hRV was the most frequently isolated pathogen (33\% of cases) in respiratory infections in pediatric cancer patients $[33.9 \%$ in acute lymphoblastic 
leukemia (ALL) and acute myeloid leukemia (AML) and 31.8\% in solid tumors] [12].

A study carried out in adult patients with hematological malignancies showed that hRV was by far the most frequently identified pathogen (in about $40 \%$ of symptomatic patients); in detail, $32 \%$ of the total patients with hRV (110) were affected by ALL or myelodysplastic syndrome (MDS), 19\% by chronic leukemia, and $49 \%$ by lymphoma, multiple myeloma (MM) or other. In addition, $45 \%$ of these patients were also HSCT recipients [33].

In HSCT patients, hRV was identified as the second most frequent cause of idiopathic pneumonia (12\% of the detected pathogens) [2]. Fisher et al. showed that hRV was the most common pathogen detected in pediatric patients who underwent HSCT, followed by PiV and RSV [34].

A retrospective study showed that around $40 \%$ of HSCT patients with documented hRV infection developed pneumonia and $60 \%$ of them had co-infections [35].

Viruses belonging to this family represent the community-acquired pathogens most frequently involved in respiratory symptoms in HSCT candidates [11].

The hRV incidence in immunocompromised children undergoing chemotherapy has been found to be up to $22.3 \%$ on the 100th day of treatment [36], and hRV is by far the most common respiratory pathogen isolated in immunocompromised children undergoing chemotherapy treatment [16].

\section{Human Metapneumovirus (hMPV)}

Human metapneumovirus is a major cause of infection in children worldwide [37]. Since it was discovered, several international studies have highlighted how primary infection occurs before 5 years of age and that all children are potentially infected at 10 years of life. Additionally, a number of reinfections may occur in subsequent years [2]. To date, we know that this pathogen can be responsible for acute respiratory pictures not only in pediatric patients, but also in elderly and immunosuppressed subjects.
A systematic review evaluating data from 17 studies on hematological patients, HSCT patients or a combination of both revealed a total incidence of hMPV infection to 5\% (range $0-40 \%$ ). The incidence observed in hematological patients $(7 \%)$ does not differ from that found in patients receiving stem cell transplantation. On the contrary, the incidence observed in adult patients was higher (7\%) than in pediatric patients (4\%). In studies in which patients with URTI and LRTI were taken into account, mortality was around 6\% (range $0-17 \%)$; however, it was significantly higher in patients with LRTI (approximately 27\%) [38]. Hakim et al. showed a $10 \%$ incidence in patients with ALL [22].

In the study conducted by Fontana and Strasfeld considering hematological and HSCT patients, an LRTI incidence between 2 and 11\% was estimated [6].

Another more recent review highlighted that $7 \%$ of all children with cancer who developed a respiratory infection were positive for hMPV. Of this $7 \%$, about $1 \%$ died; $2.3 \%$ of pediatric patients who received a transplant and were hMPV positive died as well [37].

Other studies confirm that immunocompromised children have a higher risk of developing LRTI as well as a higher risk of ICU (intensive care unit) admission and increased mortality [11].

Although hMPV generally has a self-limiting course in the general population, severe disease and fatal outcomes have been reported, especially in HSCT patients; notably, co-morbidities make establishing the clear cause of the fatal outcome difficult [2]. Compared to other viruses, hMPV infection has been found in most patients but its role in causing symptoms does not seem very crucial; nonetheless, it is not a negligible infection [11].

\section{Parainfluenza Virus (PiV)}

$\mathrm{PiV}$ is the second most frequently isolated virus as the causative agent of respiratory infections in pediatric hematological patients [39]; according to a 2016 review, ALL is the hematological neoplasm most frequently associated 
with this virus. For adults this figure is not available [40]. Fontana and Strasfeld estimate that its incidence varies between 3 and $27 \%$ in patients with hematological malignancy and/or HSCT [6]. In adults, PiV incidence ranges between 2 and 7\% [20].

According to Shah et al., the incidence in HSCT patients (4\%) is significantly higher than in hematological patients (2\%). Mortality is estimated between 10 and 50\% [6] and can reach $17 \%$ in patients with pneumonia [2]. However, no statistically significant differences between HSCT and hematological patients in terms of mortality have been highlighted [40]. Common risk factors were found to be age ( $<2$ years) and the development of LRTI.

An American study carried out in 2011 by Srinivasan et al. in pediatric patients showed that PiV caused $10 \%$ of viral respiratory infections and that PiV 3 caused most cases. PiV infections were more frequent in patients with ALL rather than in patients diagnosed with AML or lymphoma. No patients died [39].

In $20-40 \%$ of patients suffering from URTI, the infection evolves in LRTI within, on average, 78 days. PiV-related mortality is approximately $10 \%$, but increases to $27 \%$ if the infection progresses to LRTI [11].

\section{Citomegalovirus (CMV)}

CMV is an opportunistic pathogen, occasionally associated with LRTI and pneumonia, not being considered a specific respiratory virus [11].

The incidence of CMV infection in transplant patients was examined in a Chinese study. Of the total of patients (68) who underwent an allogeneic transplant, CMV infection was identified in $29 \%$ of cases, while a co-infection with RSV occurred in $26.5 \%$. The average duration of CMV infection was 60 days versus 21 days of $\mathrm{RSV}$ infection. Of the $18 \mathrm{CMV} / \mathrm{RSV}$ co-infections, 11 developed severe pneumonia (68\% of cases), while the same happened in only $31 \%$ of those who had single CMV or RSV infection. Five HSTC patients (approximately 7\%) died of severe pneumonia [17].

Seropositivity for CMV or reactivation of the same virus was documented in HSCT patients; this has been related to a greater risk of a severe clinical course and mortality [8].

\section{Bocavirus (hBoV)}

$\mathrm{hBoV}$ has been identified in samples of respiratory secretions, saliva, feces and blood in immunocompetent children. Sero-epidemiological studies showed a 90\% seropositivity within 5 years of life [15].

Bocavirus (hBoV) has been associated with $2-19 \%$ of all URTI and LRTI worldwide. The virus has also been found in patients with hematological malignancies or HSCT; however, it is difficult to establish its real impact in terms of morbidity and mortality because of the high frequency of co-infections [2]; estimated incidence ranges between 1 and 3\% [6]. A prospective study carried out on children diagnosed with cancer showed that hBoV was present in $8 \%$ of viral respiratory infections and in $19 \%$ of LRTI. In the latter, $57 \%$ of cases were related to a co-infection with other viruses [14].

\section{Adenovirus (hAdV)}

hAdV infections in HSCT recipients and in other severely immunocompromised hosts may be the consequence of new contact with the virus or the reactivation of endogenous infections [6]. In immunocompromised and HSCT pediatric recipients, hAdV is associated with severe complications and mortality. hAdV infections are less common in adult patients (incidence range: $1-30 \%)[6,11]$.

The range of hAdV-associated clinical manifestations is very wide, and URTI and LRTI are frequently caused by subgroups A, B and C. The incidence is generally higher in the pediatric population [6].

A study of adult HSTC recipients reported an infection rate of $2.5 \%$. Pneumonia occurred in $24 \%$ of cases, being the most common cause of hAdV-associated death [11].

\section{Coronavirus (CoV)}

These viruses can circulate throughout the year, with a peak in winter. Thanks to having an 
animal reservoir, to their ability to infect also humans and to quickly transmit from human to human, they are recognized as very relevant pathogens of the new century [2]. In contrast to the low incidence of bronchitis and pneumonia in healthy children, severe clinical pictures have been mainly described in immunocompromised patients [11]. A cumulative incidence of $11 \%$, 100 days after transplantation, has been shown in HSCT patients [36]. According to the study by Fontana and Strasfeld [6], the estimated incidence values range between 3 and $23 \%$ (Table 1). Given the frequency of co-infection with other viruses, the cause of death is often difficult to identify [5]. Recent studies also suggest that mortality may be comparable to that of influenza, RSV and parainfluenza virus [2].

\section{DIAGNOSTIC TOOLS}

It is not possible to reach a definite diagnosis considering only clinical manifestations; laboratory confirmation is strictly required, given that different respiratory viral infections could often have similar/overlapping clinical manifestations. Reaching a definite diagnosis is fundamental to prevent serious complications related to these infections [41].

For diagnostic purposes, culture tests, considered the gold standard, immunoassays (ELISA), direct immunofluorescence (IF) and molecular tests (PCR) are currently used. The sensitivity of any diagnostic test is strictly related to the type of sample and site of sampling. For upper respiratory tract infections, nasopharyngeal washes or aspirates or nasopharyngeal swabs, preferable to pharyngeal swabs only, are recommended [42]. In case of LRTI, tracheal aspiration or direct bronchoalveolar lavage (BAL) are recommended; the latter technique is highly sensitive but not always feasible in clinically debilitated patients [43]. However, an observational retrospective study conducted in immunocompromised patients with viral lung disease showed that the results of PCR tests performed on nasopharyngeal samples are comparable to those performed on BAL [44]. The involvement of the lower respiratory tract can be further confirmed or excluded by x-ray and computerized axial tomography (CAT) [43].

The direct immunofluorescence antigen test is a quick and inexpensive diagnostic method with variable sensitivity (range 50-93\%) [45]; however, its use does not exclude the need for infection control measures pending the results obtained by PCR [46].

The culture test is considered the gold standard for the diagnosis of these infections; however, it has a significant limitation, requiring a long time to obtain a result. Due to its high sensitivity and specificity as well as its speed, real-time polymerase chain reaction (PCR) is currently the preferred diagnostic method [5, 47].

These methods are quick, extremely sensitive and specific and have the additional advantage of being quantitative, offering the potential for multiplex testing platforms. This is a fundamental characteristic taking into account the "multi-etiological" nature of viral respiratory infections in the patients under study [48]. This feature is important for monitoring infections and for discriminating the pathogenic role of a given virus in the case of co-infections or of a low viral load. Besides, the evaluation of the viral load allows identifying patients at high risk or candidates for more urgent therapy [11]. These techniques tend to be more expensive than other direct and indirect diagnostic methods [5].

\section{THERAPEUTIC OPTIONS}

As a general rule, respiratory infection management consists of providing the patient with supportive care and, where possible, carrying out antiviral therapy [6].

For influenza, its increased resistance to M2 inhibitors (amantadine and rimantadine) has made the neuraminidase inhibitor oseltamivir the most used anti-influenza therapeutic option in immunosuppressed patients [11]. In patients with myeloma, oseltamivir reduces the risk of developing pneumonia as well as relapses [13], while a delay in the initiation of its use can lead to the onset of complications such as progression to LRTI and death in HSCT recipients [11]. 
According to the Public Health England guidance, the use of oral oseltamivir, $75 \mathrm{mg}$ twice daily for 15 days, is recommended for patients $>13$ years of age as the first-line treatment, provided that the risk of drug resistance is low. An early start of treatment, $48 \mathrm{~h}$ after the onset of symptoms in uncomplicated cases and for a minimum of 5 days, is appropriate. As an alternative therapeutic regimen, or in forms complicated by involvement of the lower respiratory tract, the use of zanamivir, in intravenous or nebulized formulation, has been approved [43].

Additional corticosteroid therapy is useful to reduce the inflammatory state and to prevent progression to LRTI; however, corticosteroids worsen the state of immunosuppression, predisposing patients to a prolongation of viral spreading [2].

Regarding RSV infection, ribavirin-based therapy has been associated with a significant reduction in the progression from URTI to LRTI [5]. Ribavirin has been proven effective in hindering the progression from URTI to LRTI (from 45 to $16 \%$ ) and in reducing mortality (from 70 to $35 \%$ ) in onco-hematological patients [5]. The lack of ribavirin aerosol therapy has increased the risk of mortality in patients with HSCT [49]; ribavirin aerosol is the only approved treatment for severe RSV-related LRTI in infants with underlying conditions such as prematurity, cardiopulmonary disease or immunosuppression [5]. In patients with myeloma, routine treatment of RSV infection is not recommended [13]. Ribavirin therapy is recommended in patients with leukemia and patients with HSCT at high risk of complications [5].

The co-administration of ribavirin with intravenous immunoglobulins (IVIG), with specific anti-RSV immunoglobulins or with pavilizumab is currently still poorly defined. However, there is a general consensus that a late start of the therapy, when pulmonary symptoms have already established, compromises its effectiveness [48]. For PiV, the use of antiviral agents such as ribavirin, intravenous immunoglobulins or a combination of these drugs does not have a significant impact on reducing the length of hospital stay, the duration of symptoms and mortality. Very few new antiviral drugs have shown promising results for the treatment of PIV infection in this type of patient [29].

A retrospective study showed that neither intravenous administration of immunoglobulins nor the use of aerosol ribavirin are useful strategies for the effective reduction of the viral load.

Therapy is mainly supportive, and since corticosteroid therapy has been shown to be a risk factor for progression towards LRTI, a valid therapeutic approach could be a reduction in the steroid dosage [43].

There is currently no specific recommended treatment for rhinovirus $[6,11]$, bocavirus [11], metapneumovirus [5] and coronavirus $[5,6,11]$.

Most patients with hMPV do not require therapy, as its efficacy in reducing the risk of progression of the infection has not been demonstrated; as asymptomatic spreading of the virus may persist, serial control by means of PCR is required [43].

Promising results following the use of IVIG and ribavirin have been obtained in vitro; however, these data have not been confirmed or validated in vivo [48].

Even for adenovirus there is no formally indicated antiviral treatment.

Several studies have been conducted with promising results in vitro regarding the use of antiviral drugs such as cidofovir, ganciclovir and other antiretroviral drugs (zalzitabine, alovudine, stavudine) but there are not as many validations in vivo [48]. Cidofovir has been shown to reduce mortality, but it is related to kidney and ocular toxicity [6].

\section{PREVENTION AND PROPHYLAXIS}

Defining effective therapeutic and prophylactic strategies for this type of infections is challenging, especially considering the spectrum variability of onco-hematological patients and the variety of respiratory viruses involved. Early diagnosis and early supportive care of high-risk patients allow reducing the progression of the pathology from the upper to lower respiratory tract [5]. 
Given the high morbidity and mortality as well as the lack of vaccines and of specific antiviral therapy for most of these infections, preventive measures remain the best approach to reducing the spread of viral infections in myeloma patients and in subjects with HSCT. It is also important to raise awareness among patients and healthcare professionals about the impact of these viruses on immunocompromised patients [29].

The reduction of transmission of viral respiratory infections is potentially feasible through the adoption of a series of measures; the most effective ones include hand hygiene, screening of visiting family members for respiratory symptoms, education of healthcare staff and isolation of the patients with respiratory symptoms $[28,43]$.

The high cost of antiviral therapy and lack of a clear efficacy in these patients highlights the need for effective vaccines against these respiratory viruses. According to the 2013 Infectious Diseases Society of America Guidelines, an intramuscular influenza vaccine is recommended for all immunocompromised patients and HSCT recipients aged 6 months or older [30]. It is also desirable to extend the annual immunization to family members and healthcare personnel in contact with the patient [43]. Even in patients with multiple myeloma, an effective preventive strategy to reduce the risk of influenza is represented by the seasonal influenza immunization. Although patients with multiple myeloma are known to not have high levels of specific anti-influenza antibodies compared to healthy subjects, the vaccine reduces the risk of URTI and the number of hospitalizations. Strategies such as immunization before transplantation or a double-dose schedule improve the immune response [13].

Neuramidase inhibitors should be prophylactically administered to all immunocompromised patients with a close contact with proven or suspected cases of infection. For influenza, the infective period is estimated from 1 day before to 7 days after the development of the symptoms, and post-exposure chemoprophylaxis should last up to 7 days after the last known exposure [4].
Regarding RSV, the prophylactic use of palivizumab (monoclonal antibody against RSV) in young children undergoing HSCT was recommended in the 2009 international guidelines to prevent infective complications; however, the lack of clear evidences on its efficacy has limited the application of this measure [29].

The use of aerosol ribavirin and pavilizumab as preventive strategies in patients with URTI evolving in LRTI is still debated, especially in lymphopenic patients [48].

Regarding $\mathrm{PiV}$, the real impact of ribavirin and of experimental anti-viral agents such as DAS 181 on mortality or on progression to pneumonia has yet to be determined [5].

Lastly, immunotherapy for RSV and influenza has so far given promising results [5].

As previously stated, infections caused by influenza virus have been successfully treated with neuramidase inhibitors (oseltamivir); however, its efficacy for influenza pneumonia has not been established, and antiviral resistance frequently occurs in immunocompromised patients.

Retrospective studies demonstrate positive outcomes with ribavirin (alone or in combination with immunomodulators) for the treatment of RSV infections in patients with hematological disease and HSCT. However, no prospective clinical studies have yet been conducted to demonstrate its clear efficacy [5].

Future studies should focus on strategies to identify patients at high risk of developing these infections and to determine the efficacy of available and new antiviral drugs.

Phase 1 studies are currently in progress to evaluate the safety and immunogenicity of a vaccine against PIV; phase 2 studies evaluating the efficacy of the new agent DAS-181 against PIV in immunocompromised patients are ongoing [13].

\section{CONCLUSIONS}

Hematological patients are by definition at risk subjects. Therefore, although respiratory viruses often cause self-limiting infections in the population, diagnosing these infections quickly in 
this group of patients is important for several reasons.

First, we need to avoid complications, even fatal ones, related to these infections [4].

Considering that the new treatments actually guarantee an increase in survival, a further positive effect could be achieved by strengthening the supportive treatments to combat these infections; this would allow obtaining better tolerability of the aggressive therapeutic regimens these patients undergo [2]. The major limitation of this review is that reported results stem from few cases, often with limited significance. However, it is confirmed that respiratory viral infections continue to cause disease in both the pre- and post-transplant period; in the event of an onset in the pre-transplant period, it is advisable to delay the transplant, but there are no clear indications in this regard. Deferral of take in charge, although it could be the most reasonable choice, is not always feasible, especially in the presence of conditions such as important lymphopenia or persistent aplasia [50].

In children, where the viral shedding is longer, delayed treatment should be strongly considered, even in asymptomatic subjects [11].

The positive outcome of the transplant itself is strongly influenced by a possible progression of the disease from the upper to the lower respiratory tract.

As mentioned, it would be useful to act on those risk factors that predispose patients to this progression. Lymphopenia appears to be the main factor implicated in this progression [11], but in preventive terms it is desirable to intervene also because of a number of other factors. It is known that the progression of the pathology is also favored by the presence of several bacterial or fungal co-infections, often transmitted by visitors and family members who have not been properly informed about the preventive measures to be taken.

In conclusion, control strategies should be implemented in the hospital environment given the limited efficacy of available therapies especially in immunocompromised subjects; preventive measures should be adopted and implemented as well [4].

\section{ACKNOWLEDGEMENTS}

Funding. No funding or sponsorship was received for the publication of this article.

Authorship. All named authors meet the International Committee of Medical Journal Editors (ICMJE) criteria for authorship for this article, take responsibility for the integrity of the work as a whole and have given their approval for this version to be published.

Disclosures. Francesco De Motoli, Federica Sandri, Maria Vittoria Toffoletto and Armando Stefanati declare that they have no conflict of interest. Giovanni Gabutti declares that he does not have a specific conflict of interest related to this paper; however, he reports grants from Sanofi Pasteur MSD, GSK Biologicals SA, Pfizer, Sanofi Pasteur Italy, MSD Italy, Emergent BioSolutions and Seqirus for taking part in advisory boards, expert meetings, acting as speaker and/or organizer of meetings/congresses and acting as principal investigator and chief of OU in RCTs.

Compliance with Ethics Guidelines. This article is based on previously conducted studies and does not contain any studies with human participants or animals performed by any of the authors.

Open Access. This article is licensed under a Creative Commons Attribution-NonCommercial 4.0 International License, which permits any non-commercial use, sharing, adaptation, distribution and reproduction in any medium or format, as long as you give appropriate credit to the original author(s) and the source, provide a link to the Creative Commons licence, and indicate if changes were made. The images or other third party material in this article are included in the article's Creative Commons licence, unless indicated otherwise in a credit line to the material. If material is not included in the article's Creative Commons licence and your intended use is not permitted by statutory regulation or exceeds the permitted use, you will need to obtain permission directly from the 
copyright holder. To view a copy of this licence, visit http://creativecommons.org/licenses/by$\mathrm{nc} / 4.0 /$.

\section{REFERENCES}

1. Vliora C, et al. A prospective study on the epidemiology and clinical significance of viral respiratory infections among pediatric oncology patients. Pediatr Hematol Oncol. 2019;36(3):173-86. https://doi. org/10.1080/08880018.2019.1613462.

2. Popescu CM, et al. Are community acquired respiratory viral infections an underestimated burden in hematology patients? Microorganisms. 2019. https://doi.org/10.3390/microorganisms7110521.

3. Girmenia C, Gentile G. Infezioni in ematologia: epidemiologia, profilassi, clinica e terapia“, cap 1 pag 1-2; Dipartimento di Biotecnologie cellulari ed ematologia, Università La Sapienza di Roma, direttori della collana Franco Mandelli, Giuseppe Avvisati, Forum Service Editore s.c.a.r.l., Genova. 1999

4. Han SB, et al. Respiratory viral infections in children and adolescents with hematological malignancies. Mediterr J Hematol Infect Dis. 2019;11(1): e2019006. https://doi.org/10.4084/mjhid.2019. 006.

5. Hijano DR, Maron G, Hayden RT. Respiratory viral infections in patients with cancer or undergoing hematopoietic cell transplant. Front Microbiol. 2018. https://doi.org/10.3389/fmicb.2018.03097.

6. Fontana L, Strasfeld L. Respiratory virus infections of the stem cell transplant recipient and the hematologic malignancy patient. Infect Dis Clin N Am. 2019;33(2):523-44. https://doi.org/10.1016/j. idc.2019.02.004.

7. Kennedy JL, Turner RB, Braciale T, Heymann PW, Borish L. Pathogenesis of rhinovirus infection. Curr Opin Virol. 2012;2(3):287-93. https://doi.org/10. 1016/j.coviro.2012.03.008.

8. Marinelli $\mathrm{T}$, et al. Respiratory viruses cause late morbidity in recipients of hematopoietic stem cell transplantation. Biol Blood Marrow Transplant. 2020. https://doi.org/10.1016/j.bbmt.2019.12.724.

9. Shi T, et al. Risk factors for respiratory syncytial virus associated with acute lower respiratory infection in children under five years: Systematic review and meta-analysis. J. Glob. Health. 2015. https:// doi.org/10.7189/jogh.05.020416.
10. Damlaj $M$, et al. Corticosteroid use as adjunct therapy for respiratory syncytial virus infection in adult allogeneic stem cell transplant recipients. Transpl Infect Dis. 2016;18(2):216-26. https://doi. org/10.1111/tid.12513.

11. Pochon C, Voigt S. Respiratory virus infections in hematopoietic cell transplant recipients. Front. Microbiol. 2019;10:1-17. https://doi.org/10.3389/ fmicb.2018.03294.

12. Aydin-Köker S, et al. A 3-year retrospective study of the epidemiology of acute respiratory viral infections in pediatric patients with cancer undergoing chemotherapy. J. Pediatr. Hematol. Oncol. 2019;41(4):e242-e246246. https://doi.org/10.1097/ MPH.0000000000001418.

13. Teh BW, Slavin MA, Harrison SJ, Worth LJ. Prevention of viral infections in patients with multiple myeloma: the role of antiviral prophylaxis and immunization. Expert Rev Anti-Infect Ther. 2015;13(11):1325-36. https://doi.org/10.1586/ 14787210.2015 .1083858 .

14. Srinivasan A, et al. Prospective detection of respiratory pathogens in symptomatic children with cancer. Pediatr. Infect. Dis. J. 2013. https://doi.org/ 10.1097/INF.0b013e31827bd619.

15. Renaud C, Campbell AP. Changing epidemiology of respiratory viral infections in hematopoietic cell transplant recipients and solid organ transplant recipients. Curr Opin Infect Dis. 2011;24(4):333-43. https://doi.org/10.1097/QCO.0b013e3283480440.

16. Loria $C$, et al. Human rhinovirus $C$ infections in pediatric hematology and oncology patients. Pediatr Transplant. 2015;19(1):94-100. https://doi.org/ 10.1111/petr.12383.

17. Yue C, et al. Virus infection facilitates the development of severe pneumonia in transplant patients with hematologic malignancies. Oncotarget. 2016;7(33):53930-40. https://doi.org/10.18632/ oncotarget.10182.

18. Hatanaka $M$, et al. Respiratory syncytial virus infection in infants with acute leukemia: a retrospective survey of the Japanese Pediatric Leukemia/ Lymphoma Study Group. Int J Hematol. 2015;102(6):697-701. https://doi.org/10.1007/ s12185-015-1890-1.

19. Kutsaya A, et al. Prospective clinical and serological follow-up in early childhood reveals a high rate of subclinical RSV infection and a relatively high reinfection rate within the first 3 years of life. Epidemiol Infect. 2016;144(8):1622-33. https://doi. org/10.1017/S0950268815003143. 
20. Bigliardi S, et al. Epidemiology and clinical outcome of lower respiratory tract infections by respiratory syncytial virus or parainfluenza virus type 3 in adults receiving treatment for either acute leukemia or severe aplastic anemia: a retrospective single center study. Ann Hematol. 2015;94(11): 1931-4. https://doi.org/10.1007/s00277-015-24553.

21. Chatzis O, et al. Burden of severe RSV disease among immunocompromised children and adults: a 10 year retrospective study. BMC Infect Dis. 2018;18(1):111. https://doi.org/10.1186/s12879018-3002-3.

22. Hakim $\mathrm{H}$, et al. Acute respiratory infections in children and adolescents with acute lymphoblastic leukemia. Cancer. 2016;122(5):798-805. https:// doi.org/10.1002/cncr.29833.

23. Benites ECA, et al. Acute respiratory viral infections in pediatric cancer patients undergoing chemotherapy. J. Pediatr. 2014;90(4):370-6. https://doi.org/10.1016/j.jped.2014.01.006.

24. Sauerbrei A, et al. Prevalence of antibodies against influenza A and B viruses in children in Germany, 2008-2010. Eurosurveillance. 2008;19(5):2014. https://doi.org/10.2807/1560-7917.ES2014.19.5. 20687.

25. Alsuwaidi AR, Al-Mekaini LA, Kamal SM, Narchi H, Souid AK. Seroprevalence of influenza A and B viruses among unvaccinated children in the United Arab Emirates: a cross-sectional study. BMC Res. 2017. https://doi.org/10.1186/s13104-017-2720-8.

26. Vilar-Compte D, et al. Influenza in patients with hematological malignancies: experience at two comprehensive cancer centers. J Med Virol. 2018;90(1):50-60. https://doi.org/10.1002/jmv. 24930.

27. Nichols WG, Guthrie KA, Corey L, Boeckh M. Influenza infections after hematopoietic stem cell transplantation: risk factors, mortality, and the effect of antiviral therapy. Clin Infect Dis. 2004;39(9):1300-6. https://doi.org/10.1086/ 425004.

28. Dykewicz CA. Hospital infection control in hematopoietic stem cell transplant recipients. Emerg Infect Dis. 2001;7(2):263-7. https://doi.org/ 10.3201/eid0702.010223.

29. Chemaly RF, Shah DP, Boeckh MJ. Management of respiratory viral infections in hematopoietic cell transplant recipients and patients with hematologic malignancies. Clin Infect Dis. 2014;59(Suppl 5): S344-S351351. https://doi.org/10.1093/cid/ciu623.
30. Cho SY, Lee HJ, Lee DG. Infectious complications after hematopoietic stem cell transplantation: current status and future perspectives in Korea. Korean J Intern Med. 2018;33(2):256-76. https://doi.org/ $10.3904 / \mathrm{kjim} .2018 .036$.

31. Tomblyn $\mathrm{M}$, et al. Guidelines for preventing infectious complications among hematopoietic cell transplantation recipients: a global perspective. Biol Blood Marrow Transplant. 2009;15(10):1143-238. https://doi.org/10.1016/j.bbmt.2009.06.019.

32. Abbas S, Raybould JE, Sastry S, de la Cruz O. Respiratory viruses in transplant recipients: more than just a cold. Clinical syndromes and infection prevention principles. Int J Infect Dis. 2017;62:86-93. https://doi.org/10.1016/j.ijid.2017.07.011.

33. Jacobs SE, et al. Clinical and molecular epidemiology of human rhinovirus infections in patients with hematologic malignancy. J Clin Virol. 2015;71:51-8. https://doi.org/10.1016/j.jcv.2015. 07.309 .

34. Fisher BT, et al. A multicenter consortium to define the epidemiology and outcomes of inpatient respiratory viral infections in pediatric hematopoietic stem cell transplant recipients. J Pediatric Infect Dis Soc. 2018;7(4):275-82. https://doi.org/10.1093/ jpids/pix051.

35. Jacobs SE, et al. Human rhinovirus infections of the lower respiratory tract in hematopoietic stem cell transplant recipients. Transpl Infect Dis. 2013;15(5):474-86. https://doi.org/10.1111/tid. 12111.

36. Milano F, et al. Human rhinovirus and coronavirus detection among allogeneic hematopoietic stem cell transplantation recipients. Blood. 2010;115(10):2088-94. https://doi.org/10.1182/ blood-2009-09-244152.

37. Martinez-Rodriguez C, del Banos-Lara M. HMPV in immunocompromised patients: frequency and severity in pediatric oncology patients. Pathogens. 2020;9(1):51. https://doi.org/10.3390/ pathogens9010051.

38. Shah DP, Shah PK, Azzi JM, El Chaer F, Chemaly RF. Human metapneumovirus infections in hematopoietic cell transplant recipients and hematologic malignancy patients: a systematic review. Cancer Lett. 2016;379(1):100-6. https://doi. org/10.1016/j.canlet.2016.05.035.

39. Srinivasan A, et al. Parainfluenza virus infections in children with hematologic malignancies. Pediatr Infect Dis J. 2011;30(10):855-9. https://doi.org/10. 1097/INF.0b013e31821d190f. 
40. Shah DP, Shah PK, Azzi JM, Chemaly RF. Parainfluenza virus infections in hematopoietic cell transplant recipients and hematologic malignancy patients: a systematic review. Cancer Lett. 2016;370(2):358-64. https://doi.org/10.1016/j. canlet.2015.11.014.

41. Choi S-M, Boudreault AA, Xie H, Englund JA, Corey L, Boeckh M. Differences in clinical outcomes after 2009 influenza A/H1N1 and seasonal influenza among hematopoietic cell transplant recipients. Blood. 2011;117(19):5050-6. https://doi.org/10. 1182/blood-2010-11-319186.

42. Hicks KL, Chemaly RF, Kontoyiannis DP. Common community respiratory viruses in patients with cancer: more than just 'common colds'. Cancer. 2003;97(10):2576-87. https://doi.org/10.1002/cncr. 11353.

43. Dignan FL, et al. BCSH/BSBMT/UK clinical virology network guideline: diagnosis and management of common respiratory viral infections in patients undergoing treatment for haematological malignancies or stem cell transplantation. Br J Haematol. 2016;173(3):380-93. https://doi.org/10.1111/bjh. 14027.

44. Lachant DJ, Croft DP, McGrane-Minton H, Prasad $\mathrm{P}$, Kottmann RM. Nasopharyngeal viral PCR in immunosuppressed patients and its association with virus detection in bronchoalveolar lavage by PCR. Respirology. 2017;22(6):1205-11. https://doi. org/10.1111/resp.13049.

45. Ray CG, Minnich LL. Efficiency of immunofluorescence for rapid detection of common respiratory viruses. J Clin Microbiol. 1987;25(2):355-7. https:// doi.org/10.1128/jcm.25.2.355-357.1987.

46. Ferguson PE, Gilroy NM, Sloots TP, Nissen MD, Dwyer DE, Sorrell TC. Evaluation of a clinical scoring system and directed laboratory testing for respiratory virus infection in hematopoietic stem cell transplant recipients. Transpl Infect Dis. 2011;13(5):448-55. https://doi.org/10.1111/j.13993062.2011.00631.x.

47. Osiowy C. Direct detection of respiratory syncytial virus, parainfluenza virus, and adenovirus in clinical respiratory specimens by a multiplex reverse transcription-PCR assay. J Clin Microbiol. 1998;36(11):3149-54. https://doi.org/10.1128/jcm. 36.11.3149-3154.1998.

48. Boeckh M. The challenge of respiratory virus infections in hematopoietic cell transplant recipients. Br J Haematol. 2008;143(4):455-67. https:// doi.org/10.1111/j.1365-2141.2008.07295.x.

49. Shah DP, et al. Impact of aerosolized ribavirin on mortality in 280 allogeneic haematopoietic stem cell transplant recipients with respiratory syncytial virus infections. J Antimicrob Chemother. 2013;68(8):1872-80. https://doi.org/10.1093/jac/ dkt111.

50. Passweg JR, Khanna N, Halter J. Respiratory viral infections prior to and after allogeneic haematopoietic cell transplantation. Br J Haematol. 2020;188(4):486-7. https://doi.org/10.1111/bjh. 16215. 